\title{
Quaderni
}

QUADERNI Communication, technologies, pouvoir

\section{Frédéric Forest, "Freud, Lacan : anatomie d'un passage. Le concept de réseau en psychanalyse"}

\section{Michel Daccache}

\section{(2) OpenEdition}

1 Journals

\section{Édition électronique}

URL : http://journals.openedition.org/quaderni/1024

DOI : 10.4000/quaderni.1024

ISSN : 2105-2956

Éditeur

Les éditions de la Maison des sciences de l'Homme

\section{Édition imprimée}

Date de publication : 5 octobre 2016

Pagination : 131-133

\section{Référence électronique}

Michel Daccache, «Frédéric Forest, "Freud, Lacan : anatomie d'un passage. Le concept de réseau en psychanalyse" », Quaderni [En ligne], 91 | Automne 2016, mis en ligne le 18 décembre 2016, consulté le 25 septembre 2020. URL : http://journals.openedition.org/quaderni/1024 ; DOI : https://doi.org/ 10.4000/quaderni. 1024 


\section{Compte rendu}

\section{Freud, Lacan : anatomie d'un passage. Le concept de réseau en psychanalyse Frédéric Forest}

Préface d'Alain Vanier, Éditions Érès/Arcanes, coll. « Hypothèses », 2015

par Michel Daccache

Université de Liège (SEEED)

1. Freud et la

Science :

éléments d'épisté-

mologie (Préface

d'Élisabeth

Roudinesco),

Paris, Économica/

Anthropos, 2010.
La question de la relation de Lacan à Freud n'a de cesse d'animer la communauté analytique. Et ce d'autant que la réponse que l'on y apporte peut se traduire non seulement aux niveaux conceptuel et théorique, mais également sur les plans thérapeutique et institutionnel. À travers cet ouvrage, F. Forest propose d'analyser l'articulation entre ces deux pensées à partir de ce qui serait leurs fondements épistémologiques cachés. Il y a quelques années, il publiait un premier ouvrage ${ }^{1}$ dans lequel il portait au jour une composante généralement ignorée de l'épistémologie freudienne, à savoir le modèle réticulaire emprunté à la neurologie qui la sous-tend de part en part. Ici, il montre que l'œuvre de Lacan s'organise elle aussi autour d'un schème réticulaire, qui fonctionnerait dès lors comme le « passeur » entre ces deux pensées.

Sur le plan conceptuel et méthodologique, F. Forest est fidèle à la démarche adoptée dans son précédent ouvrage. Il s'appuie sur la notion de réseau telle qu'appréhendée par Pierre Musso, et la développe dans le sens d'un agencement entre un « atome notionnel » (neurone pour la biologie, représentation chez Freud, signifiant chez Lacan), un flux et une fonction de régulation. L'enquête est basée sur un corpus de textes qui croise psychanalyse, histoire et épistémologie (notamment l'ensemble des œuvres publiées de Lacan). La démonstration s'articule autour de certaines œuvres ou de certains cas présentés comme « faisant date », dans la mesure où ils marqueraient une rupture dans l'histoire de la discipline tout en assurant la circulation du modèle réticulaire (alors que dans le volume précédent c'était l'Esquisse d'une psychologie scientifique de Freud qui jouait ce rôle, ici, c'est sur le cas de 
l'aphasie que l'auteur insiste). Enfin, l'iconographie occupe une place importante, les images visant à attester qu'une « raison graphique » à l'œuvre (les schémas de Saussure ou de Lacan reproduits dans l'ouvrage rendant visible la permanence d'une logique réticulaire).

F. Forest distingue ainsi trois « configurations épistémiques » correspondant à trois manières différentes d'appréhender l'esprit : l'approche neurologique (antérieure à la psychanalyse), l'approche métapsychologique (celle de Freud) et l'approche linguistique (avec Lacan). Ces trois moments de l'histoire de la discipline partagent un même schème réticulaire, qu'ils investissent cependant de manières différentes. L'approche neurologique ou physicaliste matérialise tous les éléments du réseau appréhendé comme seulement comme réseau nerveux. Freud, lui, réalise un arrachement par rapport au déterminisme biologique en développant une théorie sensible au symbolique. Ce geste est par la suite radicalisé par Lacan qui s'appuie pour cela sur la linguistique de son temps. Alors que chez Freud, les fonctions d'atome notionnel, de flux circulant et de mode de régulation sont respectivement occupées par la représentation, l'affect (ou la libido) et la dynamique plaisir-déplaisir (ou le travail d'interprétation), chez Lacan, ces places sont occupées par le Signifiant, le Désir (ou le Phallus) et le Nom-du-père (ou la scansion) (voir le tableau proposé p.130). Ces deux approches sont par ailleurs travaillées par des métaphores réticulaires renvoyant dans chaque cas à des univers différents. La théorie de Freud est habitée par un imaginaire issu de la neurologie, de l'électricité et de l'hydraulique. Lacan mobilise quant à lui un répertoire en grande partie inspiré de la linguistique saussurienne (nœud, tresse, chaîne, etc.).

L'étude de l'aphasie - qui se situe entre corps, représentation et langage-joue un rôle fondamental dans l'articulation entre ces différentes approches. Dans chacune d'elle, l'aphasie est envisagée comme " un trouble de la traduction » (p. 41). Mais le recours à la métaphore linguistique permet à Lacan de s'affranchir du biologisme. Le langage devient en effet chez Lacan « un tissage qui rend compte d'une trame, identifiée au déterminisme psychique » (p. 59). La santé mentale est alors « conçue comme libre communication et libre circulation, non pas de l'énergie comme chez Freud, mais cette fois de la parole » (p. 64). La mise au jour de la métaphore souterraine du réseau permet ainsi de rendre compte non seulement des transformations épistémologiques, mais également des évolutions dans les approches diagnostique et thérapeutique qui leur sont associées. Le durée des séances, pomme de 
discorde entre freudiens et lacaniens (temps réglementaire versus scansion) s'en trouve par là-même éclairée.

Une fois décrites ces configurations épistémiques, F. Forest va plus loin et rend compte de certains des concepts les plus fondamentaux de la pensée de Lacan à partir du modèle réticulaire. Ainsi, le sujet « sera conçu comme le lieu de la circulation de la parole ou du phallus et la métaphore du Nom-du-père appelée au rang de principe de régulation de cette circulation» (p. 19). La métaphore paternelle est, elle, envisagée comme principe de capitonnage du réseau. Et ainsi de suite. L'explicitation de l'approche lacanienne à travers la notion de réseau est développée dans le détail à travers le cas de la psychose, dont on sait qu'elle se situe au cœur de la pensée de Lacan.

Au final, F. Forest propose de répondre à une question cruciale pour la psychanalyse - le rapport de Lacan à Freud - à travers une démarche originale. L'ouvrage, érudit, permet aux spécialistes d'approfondir certaines notions ou de les découvrir sous un jour différent. Mais il peut également constituer une introduction claire à la pensée de Lacan, si difficile d'accès, pour les néophytes. Par ailleurs, F. Forest ne perd pas de vue l'un des problèmes d'actualité qui était en toile de fond de son ouvrage précédent, à savoir la montée en puissance des pratiques de soin mental basées sur des approches réductionnistes se traduisant souvent par des traitements chimiques, face auxquelles il oppose une épistémologie complexe et la cure par la parole. On peut cependant regretter que l'auteur ne fasse pas plus de place, dans la description des configurations successives, aux aspects institutionnels et sociaux indissociables des évolutions théoriques finement décrites. Il n'en apporte pas moins une importante contribution à l'épistémologie et à l'histoire de la discipline. 
